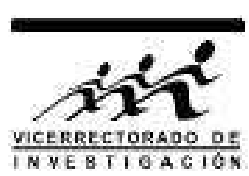

\title{
Sistemas cristalinos bidimensionales
}

\author{
D. I. Arrieta, Y. E. Huamán, M. C. Gutiérrez, R. A. Montalvo y P. H. Rivera* \\ Facultad de Ciencias Físicas, Universidad Nacional Mayor de San Marcos, Lima, Perú
}

Recibido 15 junio 2014 - Aceptado 15 julio 2014

\begin{abstract}
Desde la obtención experimental del grafeno en el 2004, los sistemas cristalinos bidimensionales han sido objeto de un profundo estudio tanto teórico como experimental. Los métodos de análisis de la estructura electrónica han abarcado desde el tight binding, la ecuación de Dirac para los puntos $\mathrm{K}$ y K' hasta los métodos de funcionales de densidad.

El presente trabajo es un estudio inicial de la estructura electrónica y la estructura de frecuencias de los fonones de los cristales bidimensionales usando la teoría de funcionales de densidad. Para ello hacemos uso de los códigos desarrollados en Fortran 90 por el Exciting y en Python por el GPAW.

Los resultados de las estructuras electrónicas obtenidos para algunas redes bidimensionales son mostrados y así como el espectro de frecuencias de los fonones del grafeno.

Palabras claves: Sistemas cristalinos 2D, teoría de funcionales de densidad, espectro de fonones, densidad de estados.
\end{abstract}

\section{Two dimensional crystalline systems}

Since the experimental obtention of graphenen in 2004, the two dimensional crystalline systems was a study subject of strong analysis from theoretical and experimental point of view. The analytical methods goes from tight binding, Dirac equations for $\mathrm{K}$ and $\mathrm{K}^{\prime}$ points to functional density theories.

The present work is an initial study of the electronic structure and the phonon frequencies spectra of 2D crystalline systems using functional density theory. We use the Exciting Fortran 90 code and GPAW Python code.

The results of electronic structure for some 2D lattices are shown and also the graphene phonon frequencies spectra.

Keywords: 2D crystalline systems, density functional theory, phonon spectra, density of states.

Los sistemas cristalinos bidimensionales son formas de cristalización periódica en la que los átomos o moléculas se distribuyen formando arreglos periódicos de largo y corto alcance en dos dimensiones, estos arreglos son definidos por el vector de traslación $t_{m}=m_{1} \boldsymbol{a}_{1}+m_{2} \boldsymbol{a}_{2}+m_{3} \boldsymbol{a}_{3}$ [1], donde $m_{1}$ y $m_{2}$ son cualquier entero positivo, negativo o cero; los vectores denotados por $\boldsymbol{a}_{1}, \boldsymbol{a}_{2}$ y $\boldsymbol{a}_{3}$ representan tres vectores arbitrarios e independientes, mientras que $m_{3}=1$ considerando que la magnitud del vector $\boldsymbol{a}_{3}$ es una longitud muy grande de modo que la interacción entre los planos establecidos por la periodicidad en la dirección $\boldsymbol{a}_{3}$ sea mucho menor que las fuerzas de van der Waals y desde este modo los planos de átomos definidos por $\boldsymbol{a}_{1}$ y $\boldsymbol{a}_{2}$ pueden ser considerados aislados uno de otro, desde el punto de vista de la simulación numérica. Pero este proceso, en la naturaleza, no minimiza la energía

*priverar@unmsm.edu.pe libre del sistema bidimensional lo que provoca inestabilidades termodinámicas que impiden su formación como red bidimensional según Landau y Peierls [2,-4]. Más aún, Mermin considera que las fluctuaciones térmicas provocan desplazamientos mayores que las distancias interatómicas que impiden la estabilidad de las estructuras bidimensionales [5]. Todas estas afirmaciones están corroboradas por una multitud de evidencias experimentales. Más aún, desde hace un tiempo, en la obtención de películas delgadas se observa que la temperatura de fusión disminuye conforme el espesor de la película disminuye [6,7], esto origina las inestabilidades termomecánicas que se contraponen a la formación de una red cristalina bidimensional.

La opción para contrarrestar estas inestabilidades es construir tales sistemas bidimensionales sobre substratos conformados por otros materiales para minimizar la ener- 
gía libre del sistema o permitiendo la formación de nanotubos [8,9] y buckybolas [10]. El primer caso fue realizado experimentalmente en el 2004 por el grupo de Andrei Geim [11] quienes consiguieron -mediante una técnica relativamente sencilla, usando una cinta adhesiva-, exfoliar capas de grafeno y colocarlas sobre un subtrato de $\mathrm{SiO}_{2}$ que se obtiene oxidando un wafer de Silicio, luego de realizar un proceso de litografías en la muestra consiguieron obtener una geometría para realizar medidas de conductividad que les permitieron deducir que el transporte de los portadores de carga se produce a través de los puntos $\mathrm{K}$ y $\mathrm{K}^{\prime}$ de la estructura electrónica proyectadas en la primera zona de Brillouin y con una velocidad de Fermi próxima al $0.3 \%$ de la velocidad de la luz en el vacío. Tales velocidades son consideradas relativísticas pero el sistema pertenece a la física de la materia condensada.

Esta noticia trajo consigo una avalancha de estudios teóricos y experimentales sobre el grafeno [12 13] y la posibilidad de obtener otros sistemas bidimensionales [14] que emulen las propiedades del grafeno o que tengan un comportamiento como semiconductor. Puesto que los portadores de carga moviéndose a velocidades consideradas relativísticas no pueden ser confinados por potenciales electrostáticos debido a la paradoja de Klein [15] que afirma que la transmisión de las partículas relativísticas a través de una barrera es igual a 1 , y esto no permitiría el uso del grafeno para la construcción de dispositivos que necesitan de un potencial confinante para discretizar las energías para determinadas aplicaciones optoelectrónicas.

Por tanto, el objetivo de este trabajo es el estudio de las propiedades electrónicas y térmicas de los cristales bidimensionales usando la teoría de funcionales de densidad para considerar los efectos de muchos cuerpos que están involucrados en la formación de la energía libre de dichos sistemas. Este trabajo es un estudio sistemático de algunos cristales bidimensionales que desde el punto de vista numérico son tratados casi aislados y a partir de los espectros de autovalores de los portadores de carga y de los iones deducimos las propiedades electrónicas y térmicas de tales sistemas.

Este trabajo consta de la primera sección que trata brevemente la evolución de los cálculos de la estructura electrónica desde Hartree hasta la teoría de funcionales de densidad como una buena aproximación al estudio de los sistemas de muchos cuerpos, y las aproximaciones que se usan en la determinación de las propiedades electrónicas y térmicas de tales sistemas. En la sección siguiente de resultados se muestran los espectros de energía y se discuten las propiedades físicas que se deducen de los análisis de los espectros. Finalmente, mostramos las conclusiones de este trabajo.

\section{Teoría}

Consideremos el hamiltoniano del sistema de muchos cuerpos como [16 17$]$

$$
\hat{H}=\hat{H}_{e}+\hat{H}_{I}+\hat{H}_{e-I}+\hat{V}_{\text {ext }}
$$

donde $\hat{H}_{e}$ es el hamiltoniano de todos los electrones que mide la energía cinética y la energía de interacción coulombiana electrón-electrón,

$$
\hat{H}_{e}=\sum_{i} \frac{\hbar^{2} \hat{k}_{i}^{2}}{2 m}+\frac{1}{2} \sum_{i \neq j} \frac{1}{4 \pi \epsilon_{0}} \frac{e_{i} e_{j}}{\left|\hat{r}_{i}-\hat{r}_{j}\right|},
$$

$\hat{H}_{I}$ es el hamiltoniano de todos los iones que mide la energía cinética y la energía de interacción coulombiana iónión,

$$
\hat{H}_{I}=\sum_{J} \frac{\hbar^{2} \hat{q}_{J}^{2}}{2 M_{J}}+\frac{1}{2} \sum_{J \neq K} \frac{1}{4 \pi \epsilon_{0}} \frac{Z_{J} e_{J} Z_{K} e_{K}}{\left|\hat{R}_{J}-\hat{R}_{K}\right|}
$$

$\hat{H}_{e_{I}}$ es el hamiltoniano de interacción coulombiana entre los iones y electrones

$$
\hat{H}_{e-I}=-\sum_{J, i} \frac{1}{4 \pi \epsilon_{0}} \frac{Z_{J} e_{J} e_{i}}{\left|R_{J}-r_{i}\right|}
$$

y finalmente, $\hat{V}_{\text {ext }}$ representa a los potenciales externos aplicados. La ecuación de autovalor de este problema está dado por

$$
\hat{H}|E\rangle=E|E\rangle
$$

donde $E$ y $|E\rangle$ representa los autovalores y los autoestados del sistema de muchos cuerpos. Este representa uno de los mayores problemas de la física porque resolver esta ecuación es prácticamente imposible con la tecnología actual que se tiene porque el número de partículas involucrada en los cálculos son del orden del número de Avogadro, $N_{a} \sim 10^{23}$ partículas.

La primera aproximación que se aplica para resolver el problema es la aproximación adiabática o la aproximación de Born-Opennheimer, que considera que el movimiento de los iones y los electrones que conforman el sólido están desacoplados porque la masa de los electrones es aproximadamente 1800 veces menor que la masa de los iones, de modo que cualquier perturbación en el movimiento de los iones los electrones siente la perturbación de manera instantánea y adiabáticamente, pero lo inverso, cualquier perturbación en el movimiento de los electrones los iones reaccionan mucho después y casi imperceptiblemente, luego los autoestados de energía del sistema de muchos cuerpos se disgrega en dos autoestados, la de los iones y 
la de los electrones, $|E\rangle=\left|E_{e}\right\rangle \otimes\left|E_{I}\right\rangle$, luego aplicando en la Ec.(5) considerando la Ec.(1) se obtiene,

$$
\begin{aligned}
& \frac{1}{\left|E_{e}\right\rangle} \hat{H}_{e}\left|E_{e}\right\rangle+\frac{1}{\left|E_{I}\right\rangle} \hat{H}_{I}\left|E_{I}\right\rangle+ \\
& \frac{1}{\left|E_{e}\right\rangle \otimes\left|E_{I}\right\rangle} \hat{H}_{e-I}\left|E_{e}\right\rangle \otimes\left|E_{I}\right\rangle+ \\
& \frac{1}{\left|E_{e}\right\rangle \otimes\left|E_{I}\right\rangle} \hat{V}_{\text {ext }}\left|E_{e}\right\rangle \otimes\left|E_{I}\right\rangle=E \\
& \Rightarrow E_{e}+E_{I}+E_{e-I}+E_{\text {ext }}=E
\end{aligned}
$$

de donde obtenemos las ecuaciones de autovalores para los electrones, iones, el acoplamiento de los iones y electrones y la acción de campos externos sobre los iones y electrones,

$$
\begin{aligned}
\hat{H}_{e}\left|E_{e}\right\rangle & =E_{e}\left|E_{e}\right\rangle \\
\hat{H}_{I}\left|E_{I}\right\rangle & =E_{I}\left|E_{I}\right\rangle \\
\hat{H}_{e-I}\left|E_{e}\right\rangle \otimes\left|E_{I}\right\rangle & =E_{e-I}\left|E_{e}\right\rangle \otimes\left|E_{I}\right\rangle \\
\hat{V}_{\text {ext }}\left|E_{e}\right\rangle \otimes\left|E_{I}\right\rangle & =V_{\text {ext }}\left|E_{e}\right\rangle \otimes\left|E_{I}\right\rangle,
\end{aligned}
$$

respectivamente.

Observando la dinámica de los iones, Ec.(8) y Ec.(9), que oscilan alrededor de un centro de equilibrio para cada uno de los iones, un primer cálculo se realiza considerando los iones estáticos en sus posiciones de equilibrio, $\hat{H}_{I}^{0}$, $\hat{H}_{e-I}^{0}$, y luego se considera la dinámica de los iones como movimientos colectivos que se manifiestan como fonones $\hat{H}_{p h}$ y estas excitaciones colectivas de los iones se acoplan al movimiento de los electrones $\hat{H}_{e-p h}$. Luego los hamiltonianos de los iones y la interacción electrón-ión se reescriben como

$$
\begin{aligned}
\hat{H}_{I} & =\hat{H}_{I}^{0}+\hat{H}_{p h} \\
\hat{H}_{e-I} & =\hat{H}_{e-I}^{0}+\hat{H}_{e-p h}
\end{aligned}
$$

de modo que se resuelve la ecuación de autovalor de manera perturbativa.

Por tanto, una buena aproximación es resolver la ecuación de autovalor considerando

$$
\begin{aligned}
& \left\langle\boldsymbol{r}\left|\left(\hat{H}_{e}+\hat{H}_{e-I}^{0}\right)\right| E_{e}\right\rangle= \\
& \left(H_{e}+H_{e-I}^{0}\right)\left\langle\boldsymbol{r} \mid E_{e}\right\rangle=E_{e}\left\langle\boldsymbol{r} \mid E_{e}\right\rangle .
\end{aligned}
$$

Hartree resuelve este problema considerando que la autofunción de muchos cuerpos y la energía de los electrones se expresa como

$$
\begin{aligned}
\left\langle\boldsymbol{r} \mid E_{e}\right\rangle & =\left\langle\boldsymbol{r}_{1} \mid E_{e_{1}}\right\rangle\left\langle\boldsymbol{r}_{2} \mid E_{e_{2}}\right\rangle \cdots\left\langle\boldsymbol{r}_{N} \mid E_{e_{N}}\right\rangle \\
& =\prod_{i=1}^{N}\left\langle\boldsymbol{r}_{i} \mid E_{e_{i}}\right\rangle \quad \text { y } \quad E_{e}=\sum_{i=1}^{N} E_{e_{i}}
\end{aligned}
$$

que al ser reemplazado en la Ec.(13) y considerando las Ecs.(2) y (4), encuentra que la minimización del valor esperado del hamiltoniano de muchos cuerpos desarrollandolo variacionalmente para determinar el estado fundamental del sistema de muchos cuerpos se consigue solo si el hamiltoniano de un electrón simple cumple la ecuación de autovalor dado por

$$
\begin{aligned}
& {\left[-\frac{\hbar^{2}}{2 m} \nabla_{i}^{2}+V\left(\boldsymbol{r}_{i}\right)+\right.} \\
& \left.\frac{e^{2}}{4 \pi \epsilon_{0}} \sum_{j \neq i} \int \frac{\left|\left\langle\boldsymbol{r}_{j}^{\prime} \mid E_{e_{j}}\right\rangle\right|^{2}}{\left|\boldsymbol{r}_{\boldsymbol{i}}-\boldsymbol{r}_{j}^{\prime}\right|} d \boldsymbol{r}_{j}^{\prime}\right]\left\langle\boldsymbol{r}_{i} \mid E_{e_{i}}\right\rangle=E_{e_{i}}\left\langle\boldsymbol{r}_{i} \mid E_{e_{i}}\right\rangle
\end{aligned}
$$

donde el primer término indica la energía cinética del electrón, el segundo término la energía potencial que los iones ejercen sobre el electrón, este término se denomina el pseudopotencial existiendo técnicas adecuadas para determinar este potencial para todos los elementos de la tabla periódica, y finalmente, el tercer término que corresponde a la interacción coulombiana electrón-electrón que se produce entre una distribución media de los electrones $j$ y el electrón $i$ que se encuentra próximo a la posición $\boldsymbol{r}_{i}$. A este término se suele denominar como el potencial de Hartree, $V^{H}$.

Existe un problema de simetría ante el operador de intercambio al considerar la autofunción de muchos cuerpos como la Ec.(14), incluyendo los autoestados de espín la autofunción de muchos cuerpos debe ser antisimétrica ante el operador de intercambio. Esta discrepancia fue resuelta por Fock al considerar la autofunción de muchos cuerpos como el determinante de Slater

$$
\left\langle\boldsymbol{r} \mid E_{e}\right\rangle=\frac{1}{\sqrt{N !}}\left(\begin{array}{cccc}
\left\langle\boldsymbol{r}_{1} \mid E_{e_{1}}\right\rangle & \left\langle\boldsymbol{r}_{1} \mid E_{e_{2}}\right\rangle & \ldots & \left\langle\boldsymbol{r}_{1} \mid E_{e_{N}}\right\rangle \\
\left\langle\boldsymbol{r}_{2} \mid E_{e_{1}}\right\rangle & \left\langle\boldsymbol{r}_{2} \mid E_{e_{2}}\right\rangle & \ldots & \left\langle\boldsymbol{r}_{2} \mid E_{e_{N}}\right\rangle \\
\vdots & \vdots & \ldots & \vdots \\
\left\langle\boldsymbol{r}_{N} \mid E_{e_{1}}\right\rangle & \left\langle\boldsymbol{r}_{N} \mid E_{e_{2}}\right\rangle & \ldots & \left\langle\boldsymbol{r}_{N} \mid E_{e_{N}}\right\rangle
\end{array}\right) .
$$

De la misma forma Fock calculó el valor esperado del hamiltoniano de muchos cuerpos, usando el método variacional para determinar la minimización del valor esperado y esto solo se consigue si el hamiltoniano de una partícula simple cumple la condición de autovalor dado por

$$
\begin{aligned}
& {\left[-\frac{\hbar^{2}}{2 m} \nabla_{i}^{2}+V\left(\boldsymbol{r}_{i}\right)+\frac{e^{2}}{4 \pi \epsilon_{0}} \sum_{j \neq i} \int \frac{\left|\left\langle\boldsymbol{r}_{j}^{\prime} \mid E_{e_{j}}\right\rangle\right|^{2}}{\left|\boldsymbol{r}_{i}-\boldsymbol{r}_{j}^{\prime}\right|} d \boldsymbol{r}_{j}^{\prime}-\right.} \\
& \left.\frac{e^{2}}{4 \pi \epsilon_{0}} \sum_{j \neq i} \int \frac{\left\langle E_{e_{j}} \mid \boldsymbol{r}_{j}^{\prime}\right\rangle\left\langle\boldsymbol{r}_{i}^{\prime} \mid E_{e_{i}}\right\rangle}{\left|\boldsymbol{r}_{i}-\boldsymbol{r}_{j}^{\prime}\right|} d \boldsymbol{r}_{j}^{\prime}\right]\left\langle\boldsymbol{r}_{i} \mid E_{e_{i}}\right\rangle=E_{e_{i}}\left\langle\boldsymbol{r}_{i} \mid E_{e_{i}}\right\rangle,
\end{aligned}
$$

respecto a la Ec.(15), los tres primeros términos son los mismos y el cuarto es la energía potencial de intercambio 
denominada como potencial de Hartree-Fock o potencial de intercambio, $V^{e x}$.

Resumiendo, la Ec.(17), para un electrón simple se expresa como

$$
\left[-\frac{\hbar^{2}}{2 m} \nabla^{2}+V(\boldsymbol{r})+V^{H}+V^{e x}\right]\left\langle\boldsymbol{r} \mid E_{e}\right\rangle=E_{e}\left\langle\boldsymbol{r} \mid E_{e}\right\rangle,
$$

esta es la ecuación que se resuelve autoconsistemente y que ha permitido el desarrollo de todos los métodos de cálculo aproximados de estructura electrónica desde los años 30s hasta los años 60s del siglo pasado.

Desde la década de 1920 existe una aproximación diferente para obtener la energía total de un sistema de muchos cuerpos considerando la densidad electrónica $n(\boldsymbol{r})$ denominada el método de Thomas-Fermi que consideraba inicialmente el sistema de electrones como un fluido clásico. En esta perspectiva, en 1964, Hohenberg y Khon [18] proponen un cálculo variacional para encontrar la energía mínima del estado fundamental de un sistema de electrones sujeto a un campo externo de iones considerando también la interacción electrón-electrón. Para ello plantean una funcional de energía en función de la densidad electrónica dado por $n(\boldsymbol{r})=\langle E \mid \boldsymbol{r}\rangle\langle\boldsymbol{r} \mid E\rangle=|\langle\boldsymbol{r} \mid E\rangle|^{2}$. Bajo este contexto, en 1965, Kohn y Sham [19] encuentran que la minimización de tal funcional exige la solución autoconsistente de ecuaciones de partícula simple que incluyen los efectos de correlación e intercambio dados por

$\left[-\frac{\hbar^{2}}{2 m} \nabla^{2}+V(\boldsymbol{r})+V^{H}+V^{x c}\right]\left\langle\boldsymbol{r}_{i} \mid E_{e_{1}}\right\rangle=E_{e_{i}}\left\langle\boldsymbol{r}_{i} \mid E_{e_{1}}\right\rangle$

y que las autofunciones de energía definen la densidad del estado fundamental como

$$
n=\sum_{i} \Theta\left(\mu-E_{e_{i}}\right)\left|\left\langle\boldsymbol{r}_{i} \mid E_{e_{i}}\right\rangle\right|^{2} .
$$

En este caso Khon-Sham encuentran el cuarto término de la Ec.(19) como el potencial de intercambio y correlación $V^{x c}$ y en la Ec.(20) la función escalera $\Theta$ asegura que los electrones ocupan los autoestados de energía sólo hasta el potencial químico (estado fundamental del sólido).

Desde esta perspectiva se define el potencial de Hartree como

$$
\begin{aligned}
V^{H}=\frac{e^{2}}{4 \pi \epsilon_{0}} \sum_{j \neq i} \int \frac{\left|\left\langle\boldsymbol{r}^{\prime} \mid E_{e_{j}}\right\rangle\right|^{2}}{\left|\boldsymbol{r}-\boldsymbol{r}^{\prime}\right|} d \boldsymbol{r}^{\prime}= \\
\frac{e^{2}}{4 \pi \epsilon_{0}} \sum_{j \neq i} \int \frac{n\left(\boldsymbol{r}^{\prime}\right)}{\left|\boldsymbol{r}-\boldsymbol{r}^{\prime}\right|} d \boldsymbol{r}^{\prime}
\end{aligned}
$$

donde la densidad incorpora los espínes $\uparrow$ y $\downarrow$ de forma

$$
\begin{aligned}
n\left(\boldsymbol{r}^{\prime}\right)=n_{\uparrow}\left(\boldsymbol{r}^{\prime}\right)+ & n_{\downarrow}\left(\boldsymbol{r}^{\prime}\right)= \\
& \left|\left\langle\boldsymbol{r}^{\prime} \mid E_{e_{i}} ; \uparrow\right\rangle\right|^{2}+\left|\left\langle\boldsymbol{r}^{\prime} \mid E_{e_{i}} ; \downarrow\right\rangle\right|^{2}
\end{aligned}
$$

y el potencial de intercambio y correlación se definen en función de la derivada de la energía total de intercambio y correlación respecto a la densidad como [20]

$$
V^{x c}=\frac{\delta E^{x c}\left[n_{\uparrow}, n_{\downarrow}\right]}{\delta n(\boldsymbol{r})}
$$

donde la energía de intercambio y correlación no se conoce de antemano, pero Kohn y Sham proponen un método que se denomina Local Density Approximation, LDA [19], que permite calcular la energía de intercambio y correlación en función de la densidad,

$$
E_{L D A}^{x c}\left[n_{\uparrow}, n_{\downarrow}\right]=\int d \boldsymbol{r}^{\prime} n\left(\boldsymbol{r}^{\prime}\right) e^{x c}\left(n_{\uparrow}, n_{\downarrow}\right),
$$

donde $e^{x c}\left(n_{\uparrow}, n_{\downarrow}\right)$ es la energía de intercambio y correlación por partícula para un gas de electrones con densidades de espínes uniformes $n_{\uparrow}$ y $n_{\downarrow}$. Este enfoque funciona muy bien para los solidos formados por los elementos de la tabla periódica que conforman el grupo I de los metales alcalinos. Con los otros elementos como por ejemplo los que poseen los orbitales hibridizados $s-p, d$ y $f$ los resultados no concuerdan con los datos experimentales.

Años más tarde, en 1996, Perdew, Burke y Ernzerhof [21] determinan la energía de intercambio y correlación con el método denominado Generalized Gradient Approximation, GGA, que involucra la dependencia de la energía de intercambio y correlación en función de la densidad y el gradiente de la densidad,

$$
E_{G G A}^{x c}\left[n_{\uparrow}, n_{\downarrow}\right]=\int d \boldsymbol{r}^{\prime} f\left(\left[n_{\uparrow}, n_{\downarrow} ; \nabla n_{\uparrow}, \nabla n_{\downarrow}\right) .\right.
$$

Este método mejora notablemente los resultados numéricos que se aproximan bastante a los datos experimentales para determinados materiales. Para otros materiales se han desarrollado funcionales para calcular la energía de correlación e intercambio que involucra una mezcla del método LDA y GGA. También existen métodos semiempíricos para LDA y GGA. Todos estos métodos están contenidos en una librería llamada libxc [22 23] que pueden ser usados por cualquier código de Density Functional Theory, DFT, nombre con el cual se ha denominado a la propuesta de Kohn, Hohemberg y Sham.

\section{Procedimiento}

Para el cálculo de las estructuras electrónicas y de los espectros de los fonones de los cristales bidimensionales hemos utilizado el código Exciting [24]. Este código usa el Fortran 90 y se basa en el método de las ondas planas linearizadas y aumentadas, Linearized Augmented Plane Waves -LAWP-, que consiste en dividir la celda unitaria en dos regiones, la primera región está ocupada por los volúmenes de los átomos conformados por esferas muffin-tin y en ella se usa como base las autofunciones de energía de 
los orbitales atómicos de cada especie atómica que contiene la celda unitaria porque el cambio de las funciones de onda en esta región varía rápidamente debido a que las autofunciones de energía son muy localizadas a distancias próximas a los átomos. Mientras, la segunda región corresponde al espacio complementario y remanente de los volúmenes ocupados por los átomos dentro de la celda unitaria denominado espacio intersticial, en ella se usan como base un conjunto de ondas planas porque las funciones de onda en esas regiones varían muy lentamente [24].

La solución autoconsistente de la Ec.(19) permite calcular la energía total del estado fundamental de los sistemas cristalinos bidimensionales. La energía de intercambio y correlación se calcula bajo la aproximación GGA [21].

\section{Propiedades electrónicas}

En esta sección mostramos los resultados de los cálculos de la estructura electrónica de las redes cristalinas bidimensionales usando la teoría de funcionales de densidad, DFT. Pero, antes mostramos la estructura electrónica del diamante.

\section{Diamante}

En primer lugar, mostramos la estructura electrónica del diamante por cuestiones metodológicas. Esto es, queremos verificar que los resultados obtenidos por el Exciting son compatibles con los resultados que se encuentran en la literatura [25]. La Figura 1 muestra la estructura electrónica del diamante, en este caso los átomos de carbono se unen via los estados hibridizados $s p^{3}$ que en intensidad energética son menores que los orbitales hibridizados $s p^{2}$. Los orbitales del diamante se distribuyen espacialmente formando dos redes cúbicas de cara centrada que se desplazan una distancia, a lo largo de la diagonal, $(1 / 4,1 / 4,1 / 4) a$ donde $a=3.567 \AA=6.740653$ Bohr es el parámetro de la red cúbica de cara centrada [26]. El máximo de la banda de valencia se encuentra en el punto $\Gamma$ y el mínimo de la banda de conducción se encuentra en la 2/3 partes del camino entre el punto $\Gamma$ y el punto $X$, con un gap indirecto de $4.088 \mathrm{eV}$, mientras que el valor experimental está en 5.47 eV a $300 \mathrm{~K}$. En la literatura sobre DFT se menciona sobre la dificultad que se tiene para calcular las estructuras tetraedrales como el diamante, la celta unitaria del diamante con los vectores $\boldsymbol{a}_{1}=(0,0.5,0.5) a$, $\boldsymbol{a}_{2}=(0.5,0,0.5) a$ y $\boldsymbol{a}_{3}=(0.5,0.5,0) a$ es un tetraedro con los átomos de carbono posicionados en los puntos $(0,0,0) a$ y $(0.25,0.25,0.25) a$. Lo que el DFT garantiza es que la banda de valencia está muy bien calculada y esto se aprecia observando la misma en la Fig 1 cuya evolución en las direcciones $\mathrm{L}, \mathrm{X}, \mathrm{W}, \mathrm{K}$ son semejantes a los encontrados en la literatura, se observan la evolución de los autoestados $s$ y $p$ ligantes que generan la banda de valencia. Para determinar, con mejor exactitud la banda de conducción, se tiene que recurrir a la teoría GW [27,28] o a la ecuación de Bethe y Salpeter [29,30] o a otros métodos como el Time Dependent Density Functional Theory, TDDFT [31] que determinan con mayor exactitud los estados excitados. En el lado derecho de la Fig. 1] se muestra de densidad de estados para todas las bandas.

El diamante es un material aislante desde el punto de vista eléctrico porque el gap es mucho mayor que las excitaciones térmicas e impiden a los electrones saltar el gap por activación térmica. La densidad de estados, DOS, es mostrada en el lado derecho de la Fig. 1 mostrando el gap indirecto.

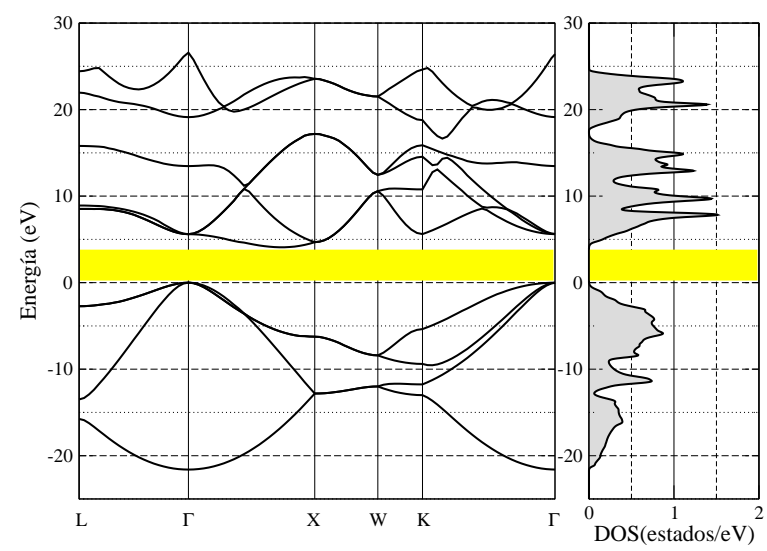

Figura 1: Se muestra la estructura electrónica y la densidad de estados del diamante obtenida mediante el Exciting.

\section{Grafito}

Para el grafito los átomos de carbono forman enlaces covalentes $s p^{2}$ en el plano $x y$ formando una estructura planar hexagonal tipo panal de abeja y en la dirección $z$ los enlaces entre los planos son de tipo van der Waals. Este tipo de interacción en $z$ produce unas propiedades mecánicas interesantes en el grafito, pues las capas de carbono son exfoliadas por un esfuerzo mecánico en la dirección $x y$ y son utilizadas ampliamente en la industria por esta propiedad como lubricantes en seco para evitar el rozamiento directo entre un par de placas metálicas y componentes de este tipo existen en casi todas las actividades industriales de la humanidad desde la automovilística hasta la aeroespacial, la seguna aplicación más difundida es el uso en los lápices que casi todo el mundo los usan desde temprana edad.

La estructura electrónica del grafito está calculada con una red unitaria definida por los vectores $\boldsymbol{a}_{1}=(1,0,0) a$, $\boldsymbol{a}_{2}=(0.5, \sqrt{3} / 2,0) a$ y $\boldsymbol{a}_{3}=(0,0,1) c / a$, donde $a=$ $\sqrt{3} d_{\mathrm{CC}}=2.464 \AA=4.6562$ Bohr, $d_{\mathrm{cC}}=1.4226 \AA=$ 2.6883 Bohr y $c=6.711 \AA=12.681952$ Bohr. Las posiciones de los átomos de carbono en la celda unitaria son $(0,0,0),(2 / 3,2 / 3,0),(1 / 3,1 / 3,1 / 2)$ y $(1,1,1 / 2)$. 
Los puntos de simetría que se muestran en la primera zona de Brillouin de la Fig. 2 pertenecen a una estructura hexagonal, asimismo se aprecia en los puntos $\mathrm{K}$ que las bandas de conducción y de valencia se degeneran lo que implica que en estos puntos el grafito es un semimetal, esa degeneración existe también en los puntos $\mathrm{H}$ cuyas coordenadas en el espacio $\boldsymbol{k}$ son $(2 / 3,1 / 3,1 / 2)$ estos puntos no están en el plano $k_{x} k_{y}$ como lo están los puntos $\mathrm{K}$ con coordenadas $(2 / 3,1 / 3,0)$. También se observa que en el punto $\Gamma$ los orbitales tipo $p$ ligantes de la banda de valencia tiene un máximo en $\sim-2.56 \mathrm{eV}$ que no es observado en los modelos tight-binding [32. En el lado derecho de la Fig. 2 se muestra la densidad de estados de todas las bandas incluyendo la degeneración en los puntos $\mathrm{K}$ y H.

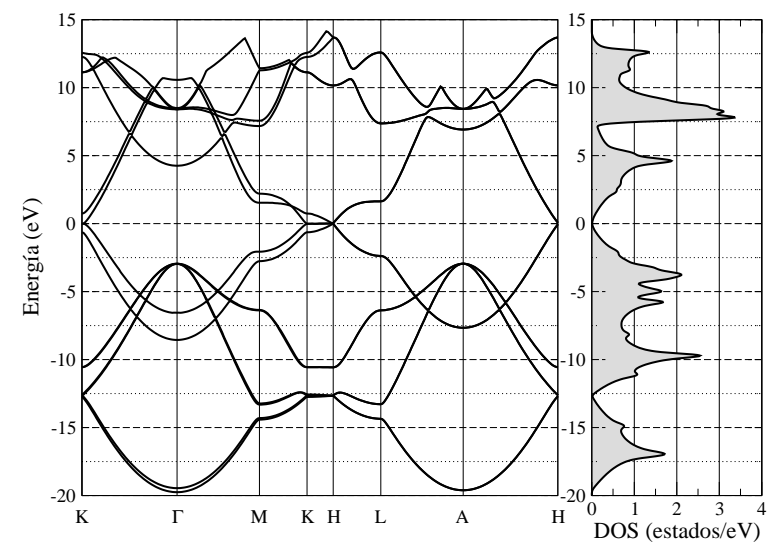

Figura 2: La estructura electrónica y la densidad de estados del grafito obtenidas mediante el Exciting son mostradas.

\section{Grafeno}

A partir de aquí, analizamos los cristales bidimensionales. La característica más importante de estos cristales son la estructura hexagonal tipo panal de abeja que se forma en el plano $x y$ formado por dos redes triangulares que se muestran en rojo y azul en el esquema que se muestra en la Fig. 3 se aprecia la celda unitaria que engloba las dos redes triangulares incorporando un átomo de cada red triangular que se unen mediante enlaces tipo $s p^{2}$ formados por los orbitales $2 p_{x}, 2 p_{y}$ y $2 s$ que se hibridizan formando los tres enlaces $\sigma$. Experimentalmente el grafeno tiene ondulaciones que dependen en algunos casos de la morfología del substrato [33] y está modulado por el orbital $2 p_{z}$ que forman enlaces tipo $\pi$ en la dirección $z$ y se adhieren al substrato mediante un potencial van der Waals.

La celda unitaria está definida por los vectores $\boldsymbol{a}_{1}=$ $(1,0,0) a, \boldsymbol{a}_{2}=(0.5, \sqrt{3} / 2,0) a$ y $\boldsymbol{a}_{3}=(0,0,0)$ con $a=$ $\sqrt{3} d_{\mathrm{cC}}=2.464 \AA=4.6562$ Bohr y $d_{\mathrm{cC}}=1.4226 \AA=$ 2.6883 Bohr. La estructura electrónica, Fig. 4 muestra que las bandas de valencia y conducción degeneran en los puntos K, estos puntos son los puntos de Dirac puesto que próximos a estos puntos la energía depende linealmente de la magnitud del vector $\boldsymbol{k} \approx(2 / 3,1 / 3,0), E_{ \pm} \approx \pm v_{F}|\boldsymbol{k}|$, y los portadores de carga se mueven a $v_{F} \sim 10^{6} \mathrm{~m} / \mathrm{s}$, esto es, $0.3 \%$ de la velocidad de la luz $c$ [34].

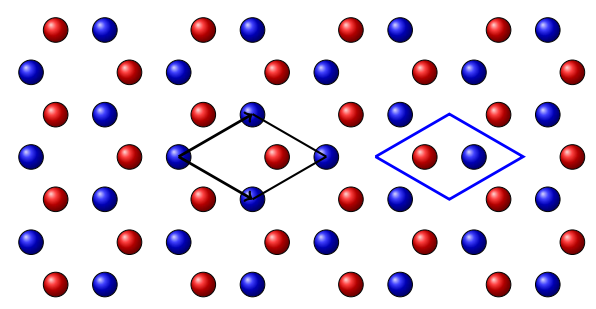

Figura 3: Esquema de las dos redes triangulares que conforman la red hexagonal tipo panal de abeja del grafeno.

Este comportamiento semimetálico con portadores de carga casi relativísticos ha despertado mucho interés en la comunidad científica. Como la paradoja de Klein [15] impide controlar el comportamiento relativístico de los portadores de carga, observando el punto $\mathrm{M}$ de la estructura electrónica se observa la posibilidad de usar el grafeno como un componente optoelectrónico porque en los puntos próximos de $\mathrm{M}$ hacia $\mathrm{K}$, las bandas de conducción y valencia forman una planicie de transición directa con posibilidad de inducir la formación de excitones con fotones de $\sim 4.5 \mathrm{eV}$ que se encuentran en la región ultravioleta, esto permitiría utilizar el grafeno en celdas solares en las regiones andinas donde existe una baja absorción de los rayos ultravioletas y en las misiones espaciales.

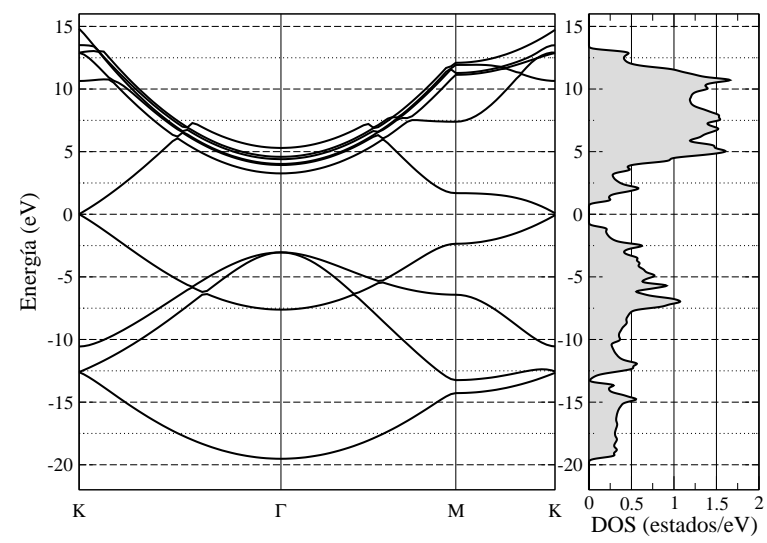

Figura 4: Se muestra la estructura electrónica y la densidad de estados del grafeno obtenida mediante el Exciting.

Por otro lado, en el lado derecho de la Fig. 4 se observa la DOS de todas las bandas que se muestran en el lado izquierdo, pero en los puntos $\mathrm{K}$, la densidad de estados no muestran el comportamiento lineal que sí se observa en el caso del grafito en los mismos puntos K, Fig. 2. Este podría ser una deficiencia del Exciting para la determinación de la DOS que se arrastraría en los otros materiales. Hemos realizado diferentes aproximaciones para el el cáluclo 
de la DOS y todas han resultado infructuosas. Los métodos tight-binding calculan mejor la DOS para los cristales bidimensionales. Para resolver este problema hemos usado también el método Grid-projector Plane Augmented Wave, GPAW [35], y hemos encontrado que mediante este método la DOS se determina relativamente bien y nos permite incorporar más bandas.

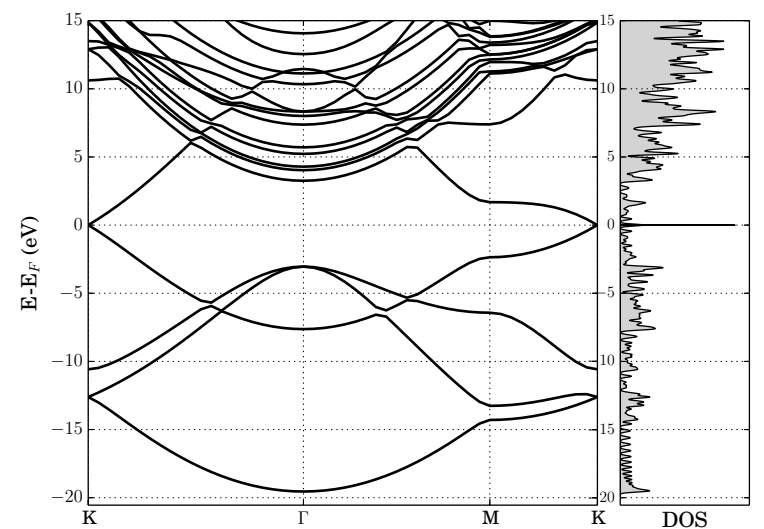

Figura 5: Se muestra la estructura electrónica y la densidad de estados del grafeno obtenida mediante el GPAW.

\section{Siliceno}

Otro cristal bidimensional que ha despertado interés en la comunidad científica es el siliceno. De manera semejante al grafeno conformado por átomos de carbono, el siliceno también tendría una estructura hexagonal tipo panal de abeja conformada por átomos de silicio, por el hecho de encontrarse el silicio debajo del carbono en la tabla periódica y podría tener la hibridización de los orbitales $3 s, 3 p_{x}$ y $3 p_{y}$ para conformar tres enlaces covalentes $s p^{2}$ -orbitales $\sigma-$, para formar la red hexagonal en el plano $x y$. En una estructura 3D, el silicio es semejante al diamante y la forma de su estructura electrónica es morfológicamente semejante al diamante [25] teniendo un gap indirecto casi en el mismo punto que el diamante, en la dirección del punto $\Gamma$ hacia y próximo al punto $\mathrm{X}$, con $E_{g}=1.1242$ eV a $T=300 \mathrm{~K}$, donde el parámetro de la red cúbica de cara centrada es $a=5.4311 \AA$ [36] 37] mayor que la del diamante, $a=3.567 \AA$.

Pero una estructura semejante al grafito, planos hexagonales con orbitales $\sigma$ en $x y$ y con orbitales $\pi$ en $z$ que permiten las interacciones van der Waals entre los planos, no existe en la naturaleza. Por ese motivo, la creación de una red planar hexagonal tipo panal de abeja se obtiene artificialmente [37] con un parámetro de red $a=3.87979$ $\AA$ y una distancia Si-Si de $2.24 \AA$.

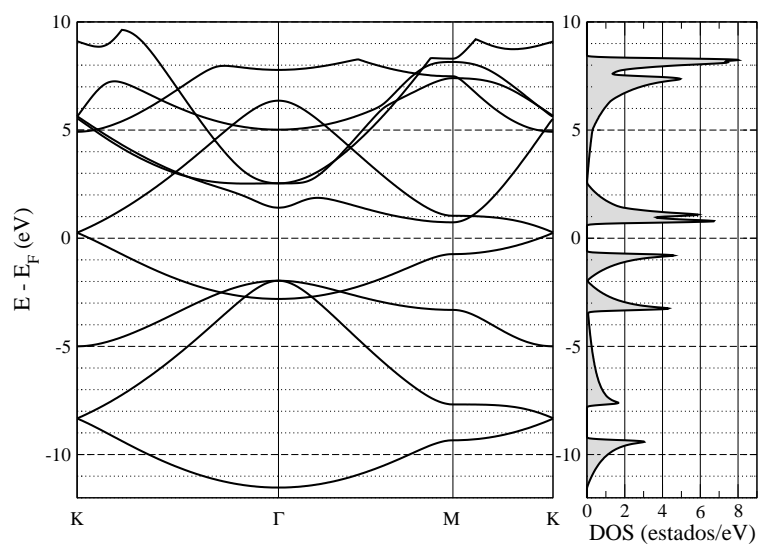

Figura 6: Se muestra la estructura electrónica y la densidad de estados del siliceno obtenida mediante el Exciting.

La estructura electrónica del siliceno obtenida mediante el Exciting se muestra en la Fig. 6y mediante el GPAW en la Fig. 7 En ambos casos se aprecia que la banda de valencia y la banda de conducción se degeneran en los puntos $X$ de forma muy semejante a la del grafeno, los puntos de Dirac, pero los anchos de las bandas de valencia son energéticamente diferentes. Con el GPAW se consigue incorporar más bandas en el cálculo poblando la banda de conducción con más autoestados, pero los modelos DFT no son muy confiables en la determinación exacta de los estados excitados como habíamos mencionado líneas atrás. Más aún si comparamos los puntos $X$ de las figuras 6 y 7 tenemos que el Exciting predice una energía de Fermi por debajo del punto $X$ lo que manifiesta un metal normal, mientras que el GPAW la energía de Fermi coincide con el punto $\mathrm{K}$ prediciendo un semimetal con portadores de carga relativísticos en el punto de Dirac, aunque la diferencia de energía entre ambos resultados es de $0.2556 \mathrm{eV}$, pero algunos experimentos confirman [37] que los portadores de carga son los fermiones de Dirac.

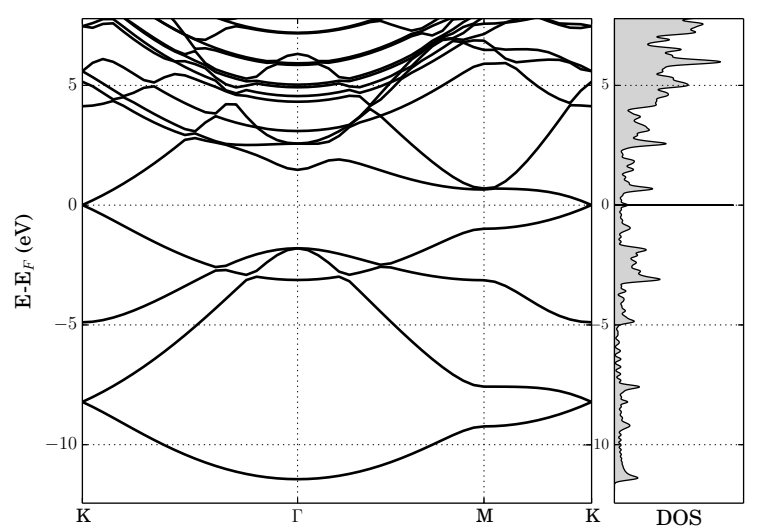

Figura 7: Se muestra la estructura electrónica y la densidad de estados del siliceno obtenida mediante el GPAW.

En el lado derecho de ambas figuras se muestra la DOS 
y las discrepancias entre ellas se ha explicado en el caso del grafeno y que se siguen manifestando en el siliceno.

También, se aprecia que en el punto $\mathrm{M}$ la probabilidad de inducir excitones con fotones de $\sim 1.47 \mathrm{eV}$ que pertenecen a la región del infrarrojo es posible. En esta región del espectro electromagnético este dispositivo tendría aplicaciones en termoterapia para ablación de células cancerígenas.

\section{Fosforeno}

Hemos afirmado en la subsección anterior que en el caso del silicio no existe un material en capas como el grafito, pero el silicio con número atómico $Z=14$ tiene como vecino al fósforo con número atómico $Z=15$ que posee varios alótropos semejantes al grafito que se caracterizan por su color -por estar relacionados con el gap entre sus bandas de valencia y conducción-, que se conocen como fósforo blanco, rojo, violeta y negro. La estructura más estable y más conocida es la del fósforo negro que tiene una estructura como se muestra en el lado izquierdo de la Fig. 8 mientras que el lado derecho muestra el fosforeno azul cuya proyección en el plano $x y$ semeja a una red hexagonal tipo panal de abeja como el grafeno [39].
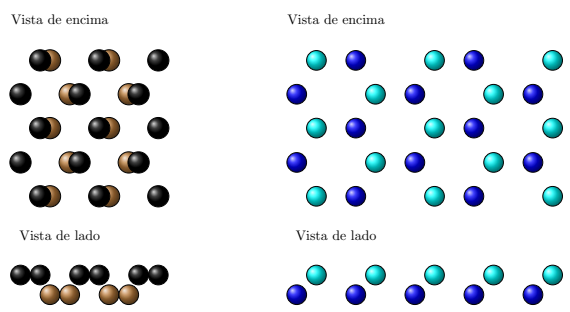

Figura 8: Se muestra la estructura de la red del fosforeno negro en el lado izquierdo y el fosforeno azul en el lado derecho.

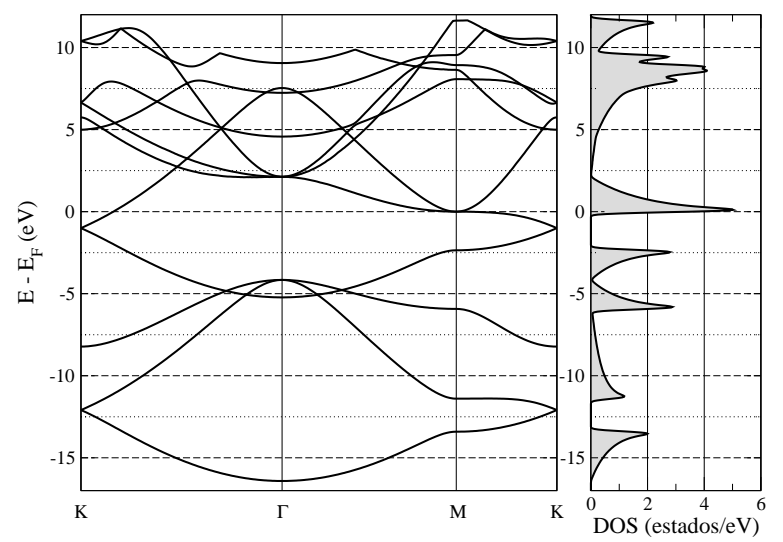

Figura 9: Se muestra la estructura electrónica y la densidad de estados de un alótropo artifical del fósforo obtenido mediante el Exciting.
En la literatura existen análisis detallados sobre el fosforeno negro 3D, mientras que el primer trabajo sobre el fosforeno azul 2D ha sido publicado recientemente [39] y así como para otros alótropos del fósforo [40]. Nuestro interés inicial es simular una red artificial hexagonal de átomos de fósforo para el cual hemos utilizado el Exciting y los resultados de esa corrida se muestra en la Fig. 9. Estamos considerando una red unitaria hexagonal con los vectores habituales $\boldsymbol{a}_{1}=(1,0,0) a, \boldsymbol{a}_{2}=(1 / 2, \sqrt{3} / 2,0) a$ y $\boldsymbol{a}_{3}=$ $(0,0,0)$ con $a=\sqrt{3} d_{\mathrm{PP}}=3.32 \AA=6.273890762$ Bohr, considerando que $d_{\mathrm{PP}}=1.9168 \AA$. Las posiciones de los átomos de fósforo respecto a los vectores de la celda unitaria son $(0,0,0)$ y $(02 / 3,2 / 3,0)$. Este espectro, Fig. 9 . tiene el mismo aspecto que las estructuras electrónicas del grafeno, Figs. 45 y del siliceno artificial, Fig. 6, en el sentido de que en los puntos $\mathrm{K}$ las bandas de valencia y conducción se degeneran, pero esta degeneración ocurre debajo de la energía de Fermi lo que significa que el fosforeno artificial sería un metal, inclusive se aprecia que en el punto $\mathrm{M}$ el nivel de Fermi intersecta la banda de conducción permitiendo que cualquier excitación térmica puede activar el transporte de electrones puesto que este fosforeno artificial es metálico desde el punto $\mathrm{K}$ hasta el punto $M$, mientras que en puntos cercanos al punto $\Gamma$ es un asilante con un gap indirecto de $\sim 2 \mathrm{eV}$.

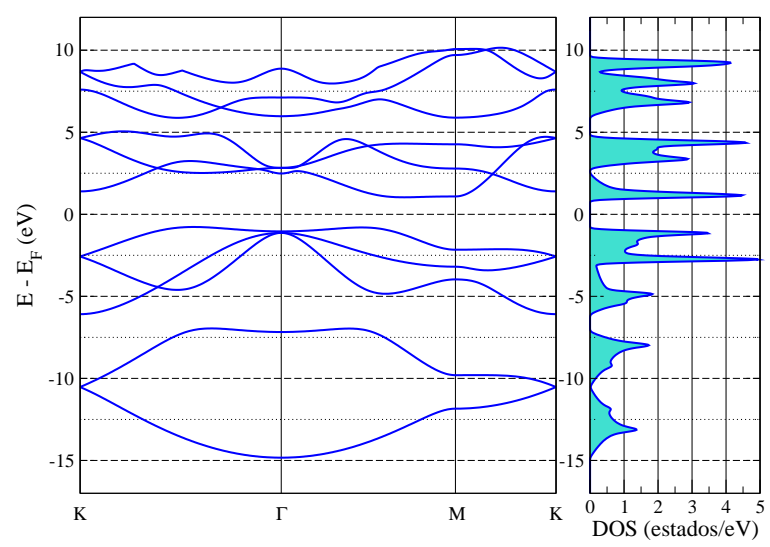

Figura 10: Se muestra la estructura electrónica y la densidad de estados del fosforeno azul obtenido mediante el Exciting.

La estructura electrónica y la densidad de estados del fosforeno azul se muestra en la figura [10 en este caso la posición del segundo átomo de fósforo cambia de $(2 / 3,2 / 3,0)$ a $(2 / 3,2 / 3,0.03163)$ y el efecto sobre la estructura electrónica es bastante sorprendente porque la figura 10 muestra a un material semiconductor de gap casi directo en un punto intermediario entre $\Gamma$ y $\mathrm{M}$. Aquí el gap es $\sim 1.8 \mathrm{eV}$, los excitones que se forman en este punto emiten fotones en la región del infrarrojo. Se observa que la banda de conducción es plana desde este punto hasta el punto M. La energía de Fermi está en el centro del gap y el eje de las energías está considerada como la diferencia 
de la energía menos la energía de Fermi. La densidad de estados muestra el gap de energía y la formación de las singularidades de Van Hove en las bandas de valencia y conducción.

\section{Molibdenita y tungstenita}

Una estructura interesante y algo compleja es la molibdenita, el disulfuro de molibdeno, $\mathrm{MoS}_{2}$. Este es un material bastante utilizado en la industria de componentes altamente tecnológicos como lubricante en seco para evitar la fricción entre dos superficies metálicas. Se utiliza desde los años 60s en la producción de armamentos y municiones avanzados. El Perú cuenta con algunos yacimientos en la Región de Huancavelica, que se explota mezclado con otros minerales como la tungstenita, $\mathrm{WS}_{2}$. Ambos materiales son conocidos desde el siglo XIX en Europa. La estructura de ambos materiales son parecidos teniendo casi las mismas dimensiones de la celda unitaria, los átomos de molibdeno (tungsteno) y los átomos de azufre forman estructuras hexagonales en planos $x y$ que están separados una distancia de $1.558 \AA$. Una monocapa de molibdenita (tungstenita) está conformado por dos capas de azufre y una capa intermedia de molibdeno (tungsteno) que tiene un corrimiento $a / 2$, donde $a=3.16$ $\AA$, desde el centro de un hexagono de átomos de azufre al centro del otro hexágono de átomos de molibdeno (tungsteno). Aquí se observa algo interesante, la proyección de los tres planos sobre el plano $x y$ forma una red hexagonal tipo panal de abeja donde los átomos de azufre forman una red triangular y los átomos de molibdeno (tungsteno) forman la otra red triangular que se asemejan al grafeno, ver la Fig. 11
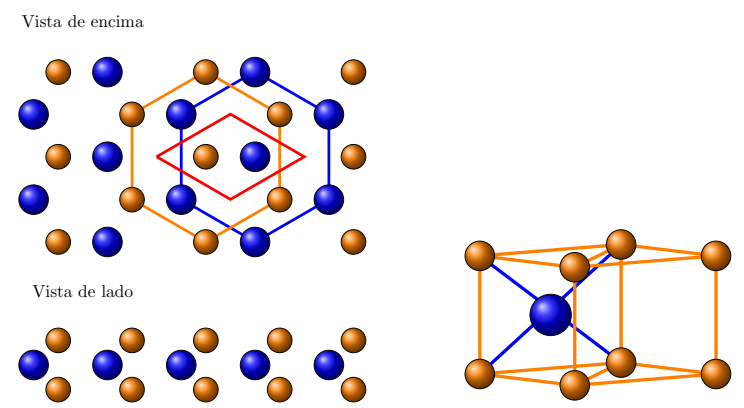

Figura 11: Se muestra la estructura de la molibdenita y la tungstenita, donde los átomos en color azul representa los átomos de molibdeno y tungsteno, respectivamente, mientras que los átomos en color anaranjado representan los átomos de azufre.

Los vectores de la celda unitaria son $\boldsymbol{a}_{1}=(1,0,0) a$, $\boldsymbol{a}_{2}=(1 / 2, \sqrt{3} / 2,0) a$ y $\boldsymbol{a}_{3}=(0,0,0.998078) c / a$, donde $a=d_{\mathrm{S}-\mathrm{S}}=d_{\mathrm{Mo}-\mathrm{Mo}}=3.122 \AA$, en el plano $x y$ y $c=$ $3.116 \AA$ para la molibdenita, mientras que $a=d \mathrm{~W}-\mathrm{W}=$ $3.155 \AA$ y $c=3.686 \AA$ para una fase y $d \mathrm{w}-\mathrm{w}=3.204 \AA$ y $c=3.763 \AA$ para una segunda fase de la tungstenita. En la Fig. 12 se muestra la estructura electrónica y la densidad de estados de la molibdenita [41] y en la Fig. 13 se muestra la estructura electrónica y la densidad de estados de la tungstenita.

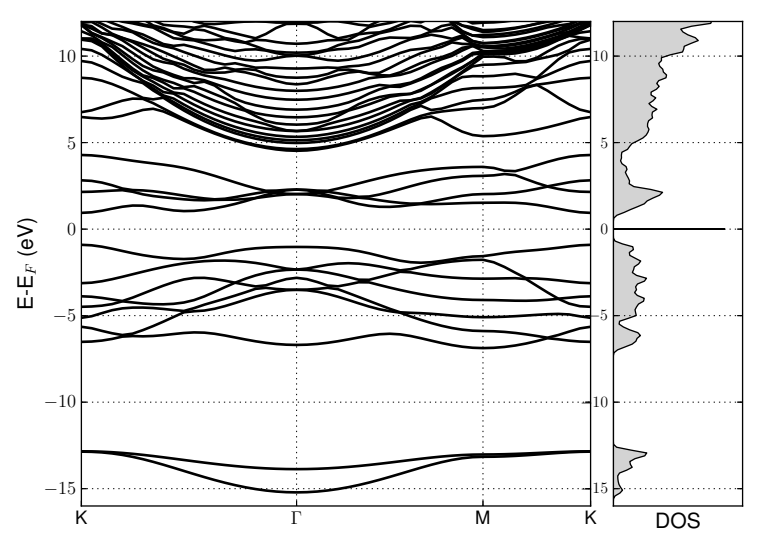

Figura 12: Se muestra la estructura electrónica y la densidad de estados de la molidenita, $\mathrm{MoS}_{2}$ obtenida mediante el GPAW.

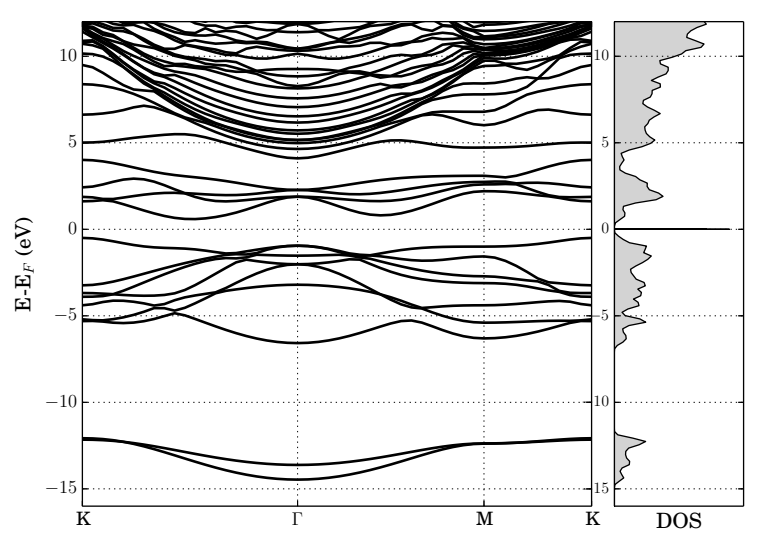

Figura 13: Se muestra la estructura electrónica y la densidad de estados de la tungstenita, $\mathrm{WS}_{2}$, fase 1 mencionada en el texto, obtenida mediante el GPAW.

Se observa que la molibdenita en la configuración estructural que se ha mencionado es un material semiconductor de gap directo en los puntos X. La densidad de estados ratifica el gap de energía de $\sim 1.74 \mathrm{eV}$. Las transiciones directas en estos puntos involucra fotones en la región del infrarrojo. En la literatura existe un trabajo realizado con el Exciting donde encuentran un gap directo en $\mathrm{X}$ de $1.79 \mathrm{eV}$ [42]. Mientras que la tungstenita muestra un semiconductor de gap indirecto de $\sim 1.0769 \mathrm{eV}$ entre el punto $X$-máximo de la banda de valencia-, y un punto intermedio entre $X$ y $\Gamma$ con un mínimo en la banda de conducción para la primera fase y para la segunda fase en el mismo punto, $\mathrm{X}$ y $\mathrm{X} \rightarrow \Gamma$ presenta un gap indirecto de $\sim 1.0606 \mathrm{eV}$. En ambos casos, las transiciones ópticas involcradas son fotones del infrarrojo. 
Los orbitales atómicos involucrados en la ligación de estos materiales son los orbitales $3 p$ del azufre y los orbitales $4 d$ del molibdeno y $5 d$ del tungsteno.

\section{Propiedades térmicas}

Analizamos las propiedades térmicas de los cristales bidimensionales a partir del espectro de frecuencias de las ramas acústicas y ópticas obtenidas mediante el Exciting. Solo presentamos el caso del grafeno y discutimos brevemente la importancia de los resultados del grafeno comparados con la del diamante y el grafito.

\section{Diamante}

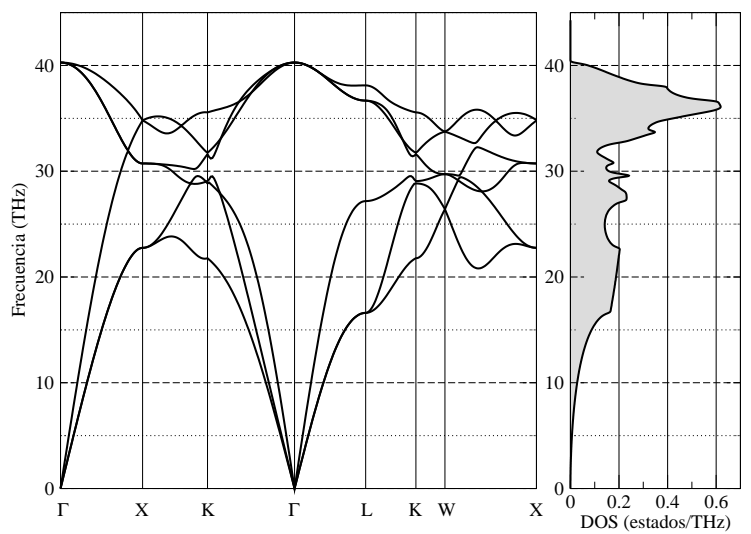

Figura 14: Se muestra el espectro de fonones y la densidad de estados del diamante obtenido mediante el Exciting.

Metodológicamente calculamos el espectro de fonones del diamante para comparar con los resultados que se encuentran en la literatura y si el Exciting reproduce dichos resultados. En la Fig. 14 mostramos el espectro de fonones y la densidad de estados de las bandas que están involucradas en las dos redes cúbicas de caras centradas desplazadas a lo largo de la diagonal y cuyas oscilaciones se muestran. Uno de los puntos experimentales que se muestran en la literatura es el máximo de la banda de frecuencias en el punto $\Gamma$ de $40 \mathrm{THz}$, y el Exciting reproduce casi exactamente este punto y la evolución de los demás puntos de simetría son congruentes con los de la literatura. Por tanto, el Exciting muestra ser una herramienta confiable para calcular el espectro de fonones para otros materiales.

\section{Grafito}

El segundo caso que analizamos con el Exciting es el caso de grafito. La Figura [15] muestra el espectro de fonones en la primera zona de Brillouin mostrando los puntos de simetría de la red hexagonal y la densidad de estados en las bandas de frecuencias del grafito.

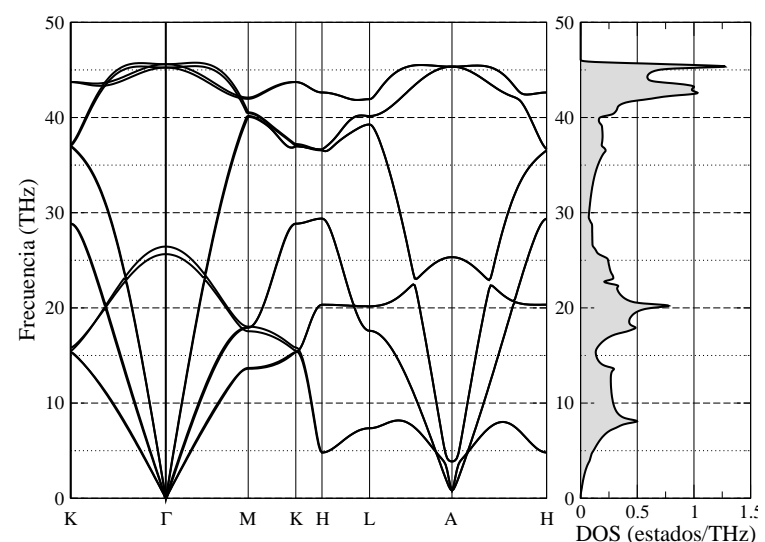

Figura 15: Se muestra el espectro de fonones y la densidad de estados del grafito obtenido mediante el Exciting.

Se observa que en el punto $\Gamma$ se encuentra las bandas máximas de frecuencias ópticas que alcanzan los $45 \mathrm{THz}$, esto suponen que el grafito poseen mejores propiedades térmicas que el diamante. Se verifica las tres ramas acústicas y las tres ramas ópticas. La primera rama óptica es menor que dos ramas acústicas en los puntos $\mathrm{K}, \mathrm{H}, \mathrm{L}$.

\section{Grafeno}

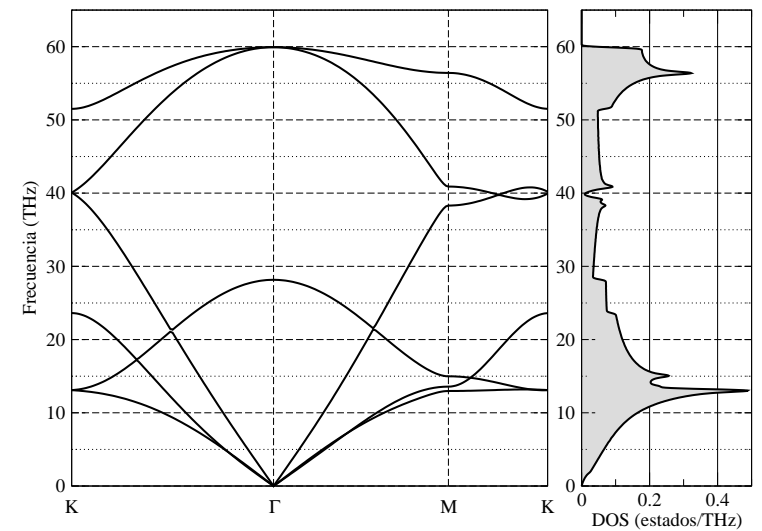

Figura 16: Se muestra el espectro de fonones y la densidad de estados del grafeno obtenido mediante el Exciting.

Los resultados que muestra el Exciting para el grafeno, Fig. 16, son bastante sorprendentes si lo comparamos con el grafito y el diamante. En primer lugar, el espectro de frecuencias presenta las tres habituales ramas acústicas y sólo dos ramas ópticas. Hay que considerar que estamos simulando solo la red hexagonal tipo panal de abeja con las dos redes triangulares mencionadas líneas atrás. No estamos considerando el substrato. El máximo de la banda 
de frecuencias ópticas se observa en el punto $\Gamma$ a la frecuencia de $60 \mathrm{THz}$. Este valor es mayor que la del grafito y la del diamante. Esto le permite al grafeno como una estructura cristalina bidimensional tener unas mejores propiedades térmicas como disipador de energía porque posee un mayor rango de frecuencias para realizar dicha tarea.

\section{Conclusiones}

En los últimos 10 años, se han estudiado los cristales bidimensionales tanto desde el punto de vista experimental así como desde el punto de vista computacional. Algunos de ellos presentan propiedades interesantes como el grafeno que posee dos puntos $\mathrm{K}$ y $\mathrm{K}^{\prime}$ donde se degeneran las bandas de valencia y conducción formando los puntos de Dirac donde los portadores de carga se comportan como fermiones de Dirac y se mueven casi relativísticamente.

Pero desde el punto de vista del control del transporte de carga, el grafeno no es un material de interés porque no presenta un gap, en cambio otros materiales como la molibdenita, el fosforeno y la tungstenita si presentan gaps directos e indirectos en regiones próximas al infrarrojo.

Esta posibilidad está alimentando el interés de la comunidad para el estudio experimental de estos cristales bidimensionales por su aplicabilidad en la producción de excitones en la región del infrarrojo que permitiría aplicaciones en biomedicina para el tratamiento del cáncer.

Hemos mostrado que el grafeno posee propiedades térmicas interesantes porque la banda de frecuencias tiene un máximo de $60 \mathrm{THz}$, un valor mayor si lo comparamos con el grafito y el diamante.

\section{Agradecimientos}

Se agradece el soporte financiero parcial al Consejo Superior de Investigaciones del Vicerrectorado de Investigación de la UNMSM mediante los proyectos $\mathrm{N}$. $^{\circ}$ s 131301081 y 141301061.

\section{Referencias}

[1] Gerald Burns, Solid State Physics, Academic Press, Inc., Orlando (1985).

[2] A. K. Geim y K. S. Novoselov; The rise of graphene, Nature Materials 6, 183 (2007).

[3] R. E. Peierls; Quelques proprietes typiques des corpses solides, Ann. I. H. Poincare 5, 177 (1935).

[4] L. D. Landau; Zur theorie der phasenumwandlungen II, Phys. Z. Sowjetunion 11, 26 (1937).

[5] N. D. Mermin; Crystalline order in two dimensions, Phys. Rev. B 176, 250 (1968).

[6] Kasturi L. Chopra; Thin film phenomena, MacGrawHill, New York (1969).

[7] J. A. Benables, G. D. T. Spiller y M. Hanbucken; Nucleation and growth of thin films, Rep. Prog. Phys. 47, 399 (1984).

[8] R. Tenne, L. Margulis, M. Genut y G. Hodes; Polyhedral and cylindrical structures of tungsten disulphide, Nature 360, 444 (1992).

[9] J. W. Mintmire, B. I. Dunlap y C. T. White; Are FuIlereue Tubules Metallic?, Phys. Rev. Lett. 68, 631 (1992).

[10] W. Krätschmer, L. D. Lamb, K. Fostiropoulos y D. R. Huffman; Solid C60: a new form of carbon, Nature 347, 354 (1990).
[11] K. S. Novoselov, A. K. Geim, S. V. Morozov, D. Jiang, Y. Zhang, S. V. Dubonos, I. V. Grigorieva y A. A. Firsov; Electric Field Effect in Atomically Thin Carbon Films, Science 306, 666 (2004).

[12] A. H. Castro Neto, F. Guinea, N. M. R. Peres, K. S. Novoselov y A. K. Geim; The electronic properties of graphene, Rev. Mod. Phys. 81, 109 (2009).

[13] A. K. Geim; Graphene: Status and Prospects, Science 324, 1539 (2009).

[14] K. S. Novoselov, D. Jiang, F. Schedin, T. J. Booth, V. V. Khotkevich, S. V. Morozov y A. K. Geim; Twodimensional atomic crystals, Proc. Nat. Acad. Sci. 102, 10451 (2005)

[15] M. I. Katsnelson, K. S. Novoselov y A. K. Geim; Chiral tunnelling and the Klein paradox in graphene, Nature Phys. 2, 620 (2006).

[16] Otfried Madelung; Introduction to Solid State Physics, Springer Verlag, Berlín (1996).

[17] R. B. Laughlin y David Pines; The Theory of Everything, Proc. Nat. Acad. Sci. 97, 28 (2000).

[18] P. Hohenberg y W. Kohn; Inhomogeneus electron gas, Phys. Rev. 136, B864 (1964).

[19] W. Kohn y L. J. Sham; Self-consistent equations including exchange and correlation effects, Phys. Rev. 149, A1133 (1965). 
[20] C. Fiolhais, F. Nogueira y M. Marques (editores); $A$ Primer in Density Functional Theory; John P. Perdew y Stefan Kurth; capítulo Density Functionals for Non-relativistic Coulomb Systems in the New Century, Springer Verlag, Berlín (2003).

[21] J. P. Perdew, K. Burke y M. Ernzerhof; Generalized Gradient Approximation Made Simple, Phys. Rev. Lett. 77, 3865 (1996).

[22] M. A. L. Marques, M. J. T. Oliveira y T. Burnus; Libxc: A library of exchange and correlation functionals for density functional theory, Comput. Phys. Commun. 183, 2272 (2012), arxiv: 1203.1739v2 (2012).

[23] http://www.tddft.org/programs/octopus/wiki /index.php/Libxc

[24] http://exciting-code.org

[25] Peter Yu y Manuel Cardona; Fundamentals of Semiconductors: Physics and Materials Properties, cuarta edición, Springer Verlag, Berlín (2010).

[26] http://www.ioffe.ru/SVA/NSM/Semicond /Diamond/index.html

[27] F. Aryasetiawan y O. Gunnarsson; The GW method, Rep. Prog. Phys. 61(3), 237 (1998). arXiv:condmat/9712013v1 (1997).

[28] Falco Hüser, Thomas Olsen y Kristian S. Thygesen; Quasiparticle GW calculations for solids, molecules, and two-dimensional materials, Phys. Rev. B 87, 235132 (2013).

[29] https://wiki.fysik.dtu.dk/gpaw /documentation/bse/bse.html

[30] http://www.berkeleygw.org/

[31] http://www.tddft.org
[32] P. R. Wallace, The Band Theory of Graphite, Phys. Rev. 71, 622 (1947).

[33] I. Pletikosić, M. Kralj, P. Pervan, R. Brako, J. Coraux, A. T. N'Diaye, C. Busse y T. Michely; Dirac Cones and Minigaps for Graphene on Ir(111), Phys. Rev. Lett. 102, 056808 (2009).

[34] A. H. Castro Neto, F. Guinea, N. M. R. Peres, K. S. Novoselov y A. K. Geim; The electronic properties of graphene, Rev. Mod. Phys. 81, 109 (2009).

[35] https://wiki.fysik.dtu.dk/gpaw/

[36] http://www.semiconductors.co.uk /propiviv5431.htm

[37] P. Vogt, P. De Padova, C. Quaresima, J. Avila, E. Frantzeskakis, M. C. Asensio, A. Resta, B. Ealet y G. Le Lay; Silicene: Compelling Experimental Evidence for Graphenelike Two-Dimensional Silicon, Phys. Rev. Lett. 108, 155501 (2012).

[38] L. Chen, C.-C. Liu, B. Feng, X. He, P. Cheng, Z. Ding, S. Meng, Y. Yao y K. Wu1; Evidence for Dirac Fermions in a Honeycomb Lattice Based on Silicon, Phys. Rev. Lett. 109, 056804 (2012).

[39] Zhen Zhu y David Tománek; Semiconducting Layered Blue Phosphorus: A Computational Study, Phys. Rev. Lett. 112, 176802 (2014).

[40] L. A. Falkovsky, Structure and electron bands of phosphorus allotropes, arXiv:cond-mat/1406.7616v1, junio (2014).

[41] K. F. Mak, Ch. Lee, J. Hone, J. Shan y T. F. Heinz; Atomically Thin $\mathrm{MoS}_{2}$ : A New Direct-Gap Semiconductor, Phys. Rev. 105, 136805 (2010).

[42] Eugene S. Kadantsev y Pawel Hawrylak; Electronic structure of a single $\mathrm{MoS}_{2}$ monolayer, Solid State Comm. 152, 909 (2012). 